\title{
MicroRNA-382 inhibits prostate cancer cell proliferation and metastasis through targeting COUP-TFII
}

\author{
WEI ZHANG ${ }^{1}$, JIANZHOU LIU ${ }^{2}$, JIANXIN QIU ${ }^{1}$, XIAOLIANG FU ${ }^{1}$, QISHENG TANG ${ }^{1}$, \\ FAN YANG $^{1}$, ZHIGUANG ZHAO ${ }^{1}$ and $\mathrm{HE} \mathrm{WANG}^{1}$ \\ ${ }^{1}$ Department of Urology, Tangdu Hospital, The Fourth Military Medical University, Xi'an, Shaanxi 710038; \\ ${ }^{2}$ Department of Urology, Central Hospital of Baoji, Baoji, Shaanxi 721008, P.R. China
}

Received May 14, 2016; Accepted September 15, 2016

DOI: $10.3892 /$ or.2016.5141

\begin{abstract}
MicroRNAs (miRNAs) have emerged as important regulators in cancer that are implicated in regulation of various cellular processes. miR-382 has been proposed as a tumor suppressor by several recent studies. However, the function of miR-382 in prostate cancer remains unknown. In this study, we aimed to investigate the potential function of miR-382 in prostate cancer. We found that miR-382 was significantly decreased in prostate cancer specimens and cancer cell lines. The overexpression of miR-382 in prostate cancer cells markedly inhibited cell proliferation, migration, and invasion. In contrast, miR-382 suppression exhibited an opposite effect. Target analysis predicted that chicken ovalbumin upstream promoter transcription factor II (COUP-TFII) was a direct target of miR-382. This prediction was experimentally confirmed by dual-luciferase reporter assay, real-time quantitative polymerase chain reaction, and western blot analysis. Our results further demonstrated that miR-382 inhibited the downstream genes of COUP-TFII, including Snail and matrix metalloproteinase 2 (MMP2). Moreover, the restoration of COUP-TFII expression significantly blocked the inhibitory effect of miR-382 on cell proliferation, migration, and invasion, and Snail expression. Taken together, this study suggests that miR-382 inhibits prostate cancer cell proliferation and metastasis through inhibiting COUP-TFII, representing an important new mechanism for understanding prostate cancer
\end{abstract}

Correspondence to: Dr Zhiguang Zhao or Dr He Wang, Department of Urology, Tangdu Hospital, The Fourth Military Medical University, 569 Xinsi Road, Baqiao, Xi'an, Shaanxi 710038, P.R. China

E-mail: zhaozhiguang118@sina.com

E-mail: wanghe_td@sina.com

Abbreviations: miRNAs, microRNAs; COUP-TFII, chicken ovalbumin upstream promoter transcription factor II; UTR, untranslated region; MMP2, matrix metalloproteinase 2

Key words: prostate cancer, miR-382, COUP-TFII, cell proliferation, metastasis pathogenesis and providing a novel therapeutic candidate target for prostate cancer therapy.

\section{Introduction}

Prostate cancer is one of the most common malignancies in men, representing the second leading cause of cancer-related mortality (1). In spite of current advances in cancer therapies, prostate cancer remains an intractable disease because of its malignant proliferation, high metastatic feature, and frequent relapses (2). However, the mechanism of prostate cancer tumorigenesis is still not fully understood. Therefore, there is an imperative need to explore new and potential therapeutic targets associated with prostate cancer malignant proliferation and high metastasis.

MicroRNAs (miRNAs) are a family of endogenous, small non-coding RNAs that participate in various cellular processes (3). miRNAs function through targeting the 3'-untranslated region (UTR) of target messenger RNA (mRNA) that negatively regulates gene expression $(4,5)$. Increasing evidence reveals that a variety of miRNAs are dysregulated in numerous types of cancers, functioning as either oncogenes or tumor suppressors $(6,7)$. miRNAs have been suggested to exhibit potential value in cancer diagnosis, prognosis, and treatment $(3,8)$. miRNAs perform important functions in the pathogenesis of prostate cancer (9). However, the precise role of miRNAs in prostate cancer remains largely unknown.

Chicken ovalbumin upstream promoter transcription factor II (COUP-TFII), also known as nuclear receptor 2 family 2 , is an important transcriptional factor that belongs to the steroid/thyroid hormone receptor superfamily (10). COUP-TFII performs a critical function in regulating various biological processes, including angiogenesis, organ genesis, neuronal development, cardiovascular development, and metabolic homeostasis (11-14). Thus, the dysregulated COUP-TFII contributes to numerous pathological processes, especially cancer $(15,16)$. Increasing number of studies has demonstrated that COUP-TFII is overexpressed in numerous types of cancers, including lung (17), colorectal (18), pancreatic (19), and prostate cancers (20). COUP-TFII performs an important function in regulating cancer microenvironment, cell growth and proliferation, metastasis, and drug resistance 
$(16,21)$. These findings highlight that COUP-TFII is a potential molecular target for anticancer interventions.

Recent studies have reported that miR-382 may function as a tumor suppressor in colorectal cancer (22), ovarian cancer (23), esophageal squamous cell carcinoma (24), and osteosarcoma (25). However, whether miR-382 functions in prostate cancer remains unknown. In this study, we aimed at investigating the potential function of miR-382 in prostate cancer. We showed that the expression of miR-382 was significantly downregulated in prostate cancer tissues and cell lines. The overexpression of miR-382 markedly inhibited prostate cancer cell proliferation, migration, and invasion. Furthermore, we predicted the targets of miR-382 by bioinformatic algorithms and verified that COUP-TFII represented a functional target gene in prostate cancer. This study demonstrates that the interference of COUP-TFII expression by miR-382 is a promising therapeutic strategy for prostate cancer treatment.

\section{Materials and methods}

Clinical specimens. Fifteen paired prostate malignant cancer tissues and adjacent normal prostate tissues were obtained from prostate cancer patients who underwent resection operation in Xi'an Tangdu Hospital. Written informed consent was obtained from the patients, and the study was approved by Institutional Human Experiment and Ethics Committee of Xi'an Tangdu Hospital.

Cell lines and cell culture. Human prostate cancer cell lines (LNCaP, PC-3, 22RV-1 and DU-145), normal prostate epithelial cells (RWPE-1), and 293T cells were purchased from American Type Culture Collection (Manassas, VA, USA). Prostate cancer cell lines and 293T cells were grown in RPMI-1640 and Dulbecco's modified Eagle's medium, respectively, all supplemented with $10 \%$ fetal bovine serum (FBS), $100 \mathrm{mg} / \mathrm{ml}$ streptomycin and $100 \mathrm{U} / \mathrm{ml}$ penicillin (all reagents from Gibco, Rockville, MD, USA). RWPE-1 cells were grown in keratinocyte serum-free media supplemented with $5 \mathrm{ng} / \mathrm{ml}$ human recombinant epidermal growth factor, $0.05 \mathrm{mg} / \mathrm{ml}$ bovine pituitary extract, $100 \mathrm{mg} / \mathrm{ml}$ streptomycin, and $100 \mathrm{U} / \mathrm{ml}$ penicillin (all reagents obtained from Gibco). All cells were cultured in a humidified incubator containing $5 \% \mathrm{CO}_{2}$ at $37^{\circ} \mathrm{C}$.

Cell transfection. The miR-382 mimics, antisense nucleotides of miR-382 (anti-miR-382), and control oligonucleotides (Ctrl. Oligo) were purchased from Life Technologies (Carlsbad, CA, USA). Prostate cancer cells were transiently transfected with 50 nM miRNA using Lipofectamine 2000 (Life Technologies) according to the manufacturer's instructions. After incubated for $48 \mathrm{~h}$, transfection efficiency was detected by real-time quantitative polymerase chain reaction (RT-qPCR) analysis.

$R T-q P C R$. Total RNA was extracted using TRIzol reagent (Life Technologies) according to the recommended protocols of the manufacturer. A total of $500 \mathrm{ng}$ total RNA was used for the reverse transcription of cDNA and miRNA by M-MLV reverse transcriptase (Clontech, Palo Alto, CA, USA) and miScript Reverse Transcription kit (Qiagen, Dusseldorf, Germany), respectively. The mRNA and miRNA transcripts were quantified by SYBR Premix Ex Taq GC kit (Takara,
Dalian, China) on an ABI7500 real-time PCR detection system (Applied Biosystems, Carlsbad, CA, USA). Glyceraldehyde-3phosphate dehydrogenase (GAPDH) or U6 small nuclear RNA was used as the internal reference. The relative gene expression was normalized against internal reference gene expression by $2^{-\Delta \Delta C t}$ method. Fold changes in gene expression were obtained by normalization with the control group.

3-(4,5-dimethyl-thiazol-2-yl)-2,5-diphenyltetrazolium bromide (MTT) assay. Cells were plated in 96-well plates at a density of $1 \times 10^{4}$ cells/well and cultured for $24 \mathrm{~h}$. Cells were transfected with miR-382 mimics or anti-miR-382 for 24, 48 and $72 \mathrm{~h}$. Then, $20 \mu \mathrm{l}$ of MTT solution $(5 \mathrm{mg} / \mathrm{ml}$; Sigma, St. Louis, MO, USA) was added to each well and incubated for $4 \mathrm{~h}$. The medium was discarded, and the formazan crystal was dissolved by adding $200 \mu \mathrm{l}$ dimethyl sulfoxide (Sigma) per well. The absorbance at a wavelength of $490 \mathrm{~nm}$ was detected by a microplate spectrophotometer (Bio-Tek Instruments, Winooski, VT, USA).

Colony formation assay. Cells were seeded into 6-well plates and transfected with miR-382 mimics or anti-miR-382. After $48 \mathrm{~h}$, the transfected cells $(200$ cells/well) were cultured in growth medium containing $0.3 \%$ noble agar to allow formation of natural colonies. After 12 days, the plates were stained with crystal violet (Sigma) in $70 \%$ ethanol. The number of stained colonies was counted under a microscope (Olympus, Tokyo, Japan).

Cell migration assay. Cell migration assay was performed using a 24-Transwell plate (Corning Inc., Corning, Toledo, NY, USA). In brief, cells were transfected with miR-382 mimics or anti-miR-382 for $48 \mathrm{~h}$, and then starved overnight in serum-free medium. Then, $200 \mu \mathrm{l}$ serum-free medium containing $1 \times 10^{4}$ transfected cells were seeded into the upper chamber, and $500 \mu \mathrm{l}$ growth medium containing $10 \%$ FBS was added in the lower chamber. After incubation for $20 \mathrm{~h}$ at $37^{\circ} \mathrm{C}$, cells that had migrated to the lower filter side were stained with $0.1 \%$ crystal violet (Sigma). The number of migrated cells was counted under a microscope (Olympus).

Cell invasion assay. For the detection of cell invasion ability, the undersurface of the Transwell filter was precoated with Matrigel (BD Bioscience, San Jose, CA, USA). The transfected cells $\left(1 \times 10^{4}\right.$ cells) in $200 \mu$ of serum-free medium were added to the upper chamber, and $500 \mu \mathrm{l}$ of growth medium containing $10 \%$ FBS was added in the lower chamber as a chemoattractant. After incubation for $24 \mathrm{~h}$ at $37^{\circ} \mathrm{C}$, the invaded cells on in the lower filter side were stained with $0.1 \%$ crystal violet (Sigma) and counted under the microscope (Olympus).

Dual-luciferase reporter assay. The 3'-UTR of COUP-TFII matching miR-382 sequences were cloned into pmirGLO dualluciferase vector (Promega, Madison, WI, USA) downstream of the luciferase open reading frame. The mutant 3'-UTR of COUP-TFII containing the mutation of miR-382 recognition sites was synthesized using a Site-Directed Mutagenesis kit (Agilent Technologies, Santa Clara, CA, USA) and cloned into the pmirGLO dual-luciferase vector (Promega). The 293T cells were cotransfected with miR-382 mimics and the 
A

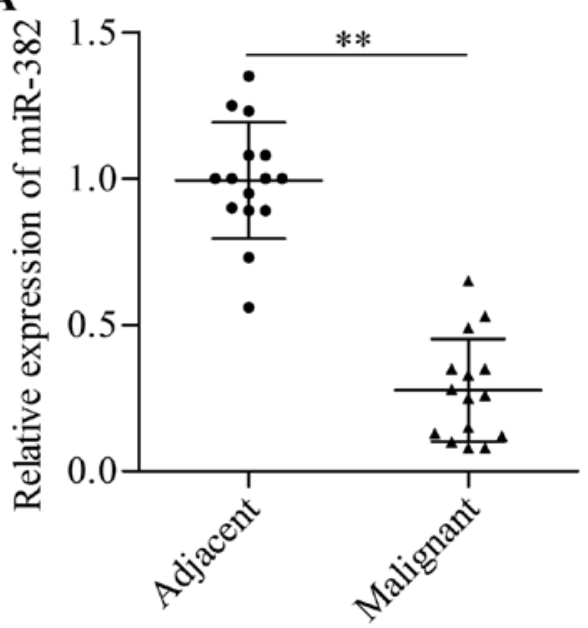

B

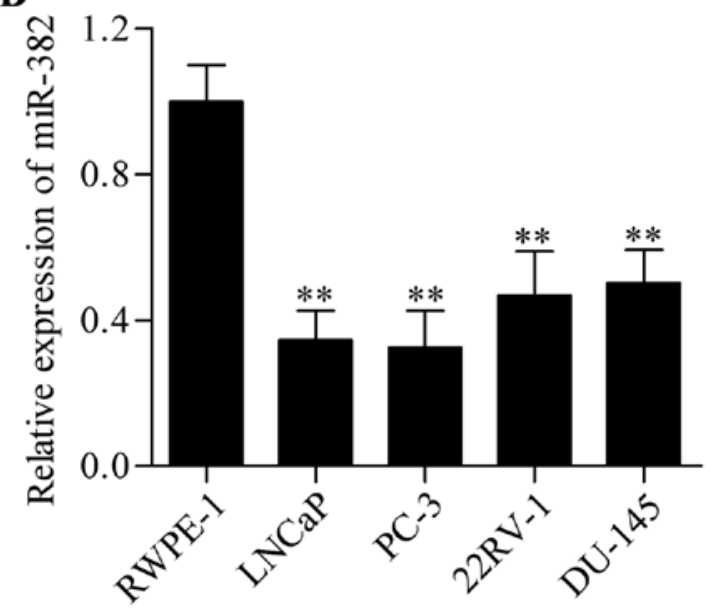

Figure 1. Decreased expression of miR-382 in prostate cancer. (A) The expression of miR-382 in 15 paired prostate malignant cancer tissues and adjacent normal prostate tissues was examined by RT-qPCR analysis. ${ }^{* *} \mathrm{p}<0.01$ vs. adjacent. (B) The expression of miR-382 in prostate cancer cell lines (LNCaP, PC-3, 22RV-1, and DU-145) and normal prostate RWPE-1 cells was detected by RT-qPCR analysis. ${ }^{* *}$ p $<0.01 \mathrm{vs.} \mathrm{RWPE-1.}$

pmirGLO vector. Luciferase assay was performed using a dual-Glo luciferase assay system (Promega).

Western blot analysis. Equal amounts of proteins from different samples were subjected to sodium dodecyl sulfate polyacrylamide gel electrophoresis. The separated proteins were then transferred to a polyvinylidene difluoride membrane (Millipore, Boston, MA, USA). The membrane was blocked by $3 \%$ non-fat milk in Tris-buffered saline (TBS) for $1 \mathrm{~h}$ at $37^{\circ} \mathrm{C}$. Then, the membrane was incubated with primary antibodies in TBS at $4^{\circ} \mathrm{C}$ overnight. After three washes with TBS containing $0.1 \%$ Tween-20, the membrane was incubated with horseradish peroxidase-conjugated secondary antibodies (1:2,000; Santa Cruz Biotechnology, Santa Cruz, CA, USA) at room temperature for $4 \mathrm{~h}$. Protein bands were detected by chemiluminescence (Pierce, Woburn, MA, USA). Protein expression was quantified using Image-Pro Plus 6.0 software (Media Cybernetics, Inc., Rockville, MD, USA) normalized against internal control (GAPDH). Anti-COUP-TFII (ab42672; 1:250) was purchased from Abcam (Cambridge, UK). Anti-Snail (sc-28199; 1:200), anti-MMP2 (sc-10736; 1:200), and anti-GAPDH (sc-25778; 1:500) were obtained from Santa Cruz Biotechnology.

Statistical analysis. All numerical data were expressed as mean \pm standard deviation. Statistical analyses were performed using the commercial statistical software, SPSS version 11.5 (SPSS Inc., Chicago, IL, USA). Differences between two groups were analyzed by a paired two-tailed Student's t-test. Data of more than two groups were analyzed using one-way analysis of variance followed by a Bonferroni post-analysis. $\mathrm{p}<0.05$ was considered statistically significant.

\section{Results}

Expression of miR-382 is decreased in prostate cancer. To investigate the potential function of miR-382 in prostate cancer, we examined the expression in prostate cancer clinical specimens by RT-qPCR. Interestingly, our data showed that
miR-382 was significantly decreased in prostate cancer tissues compared with adjacent normal prostate tissues (Fig. 1A). In addition, we found that the expression of miR-282 was markedly decreased in human prostate cancer cell lines compared with normal prostate RWPE-1 cells (Fig. 1B). Taken together, our data suggest that miR-382 may perform an important function in the development and progression of prostate cancer.

miR-382 inhibits prostate cancer cell proliferation. To investigate the function of miR-382 in prostate cancer, we performed gain-of-function and loss-of-function experiments by transfection of miR-382 mimics and anti-miR-382 in prostate cancer cell lines, respectively, and detected their effect on cell proliferation. MTT assay showed that the overexpression of miR-382 significantly inhibited prostate cancer cell proliferation, but the inhibition of miR-382 significantly increased prostate cancer cell proliferation (Fig. 2A and B). Furthermore, the colony-forming capacity of prostate cancer cells was markedly suppressed by miR-382 overexpression whereas suppression of miR-382 showed an opposite effect (Fig. 2C and D). These results indicate that miR-382 performs an antitumor function in prostate cancer.

miR-382 inhibits prostate cancer cell metastasis in vitro. To further evaluate the biological function of miR-382 in prostate cancer, we detected the function of miR-382 in regulating prostate cancer cell migration and invasion. The results showed that the overexpression of miR-382 significantly inhibited LNCaP and PC3 cell migration (Fig. 3A) and invasion (Fig. 3B) in vitro. Conversely, the inhibition of miR-382 markedly promoted the migration and invasion of $\mathrm{LNCaP}$ and PC3 cells (Fig. 3). These data imply that miR-382 performs an anti-metastatic function in prostate cancer.

miR-382 directly targets COUP-TFII in prostate cancer cells. To understand the underlying mechanism of miR-382 in exerting its function in regulating cell proliferation and metastasis of prostate cancer, we used algorithms to predict 

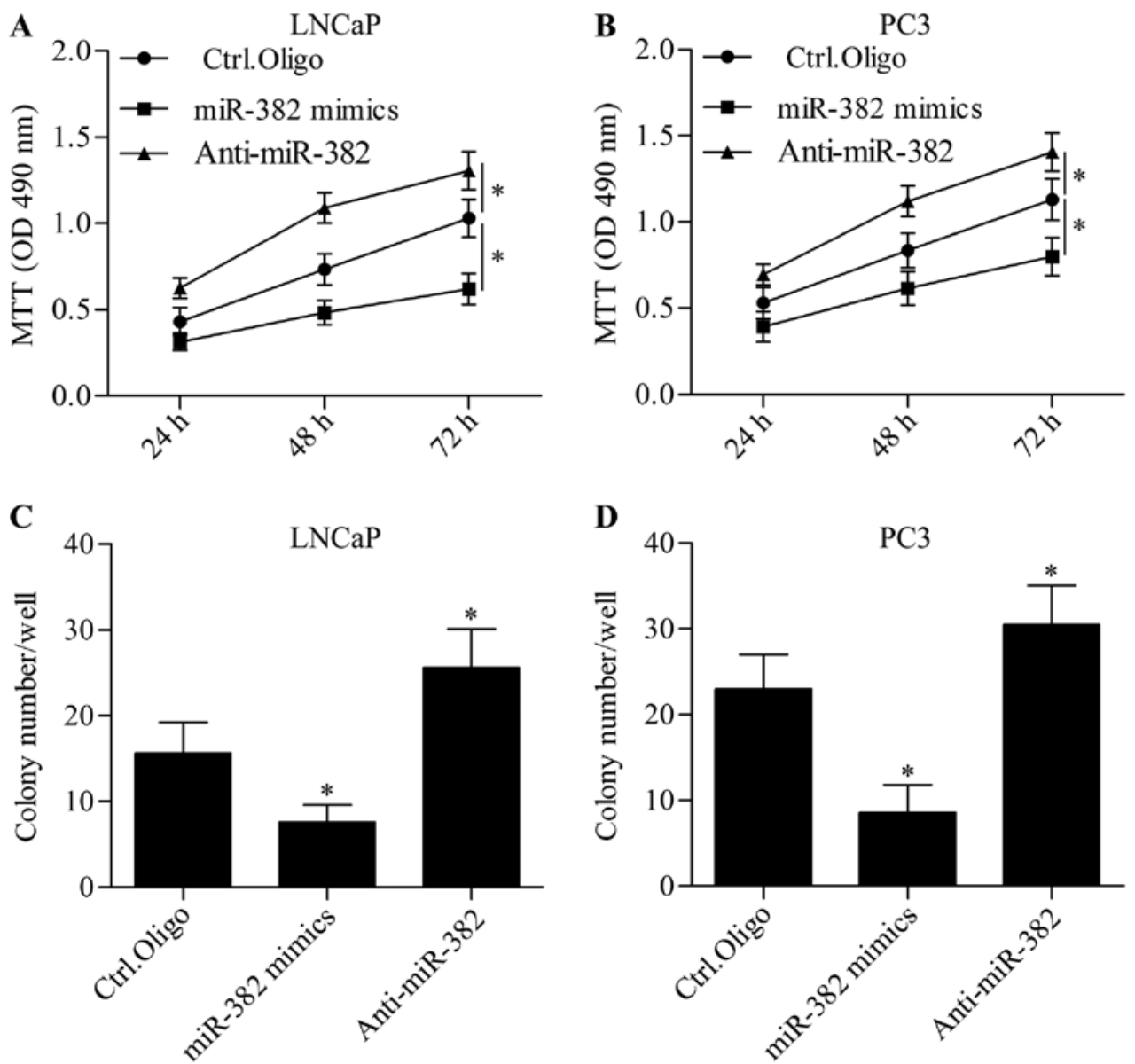

Figure 2. miR-382 inhibits prostate cancer cell proliferation. Prostate cancer cells were transfected with miR-382 mimics or anti-miR-382. MTT assay of the effect of miR-382 mimics and anti-miR-382 on cell proliferation of LNCaP (A) and PC3 (B) cells at 24, 48 and $72 \mathrm{~h}$. "p<0.05 vs. Ctrl.Oligo. The effect of miR-382 mimics and anti-miR-382 on the colony-forming capacity of LNCaP (C) and PC3 (D) cells was detected by colony formation assay. After 12 days, colonies were stained with crystal violet, and the colony number was counted under the microscope. ${ }^{*} \mathrm{p}<0.05$ vs. Ctrl. Oligo.
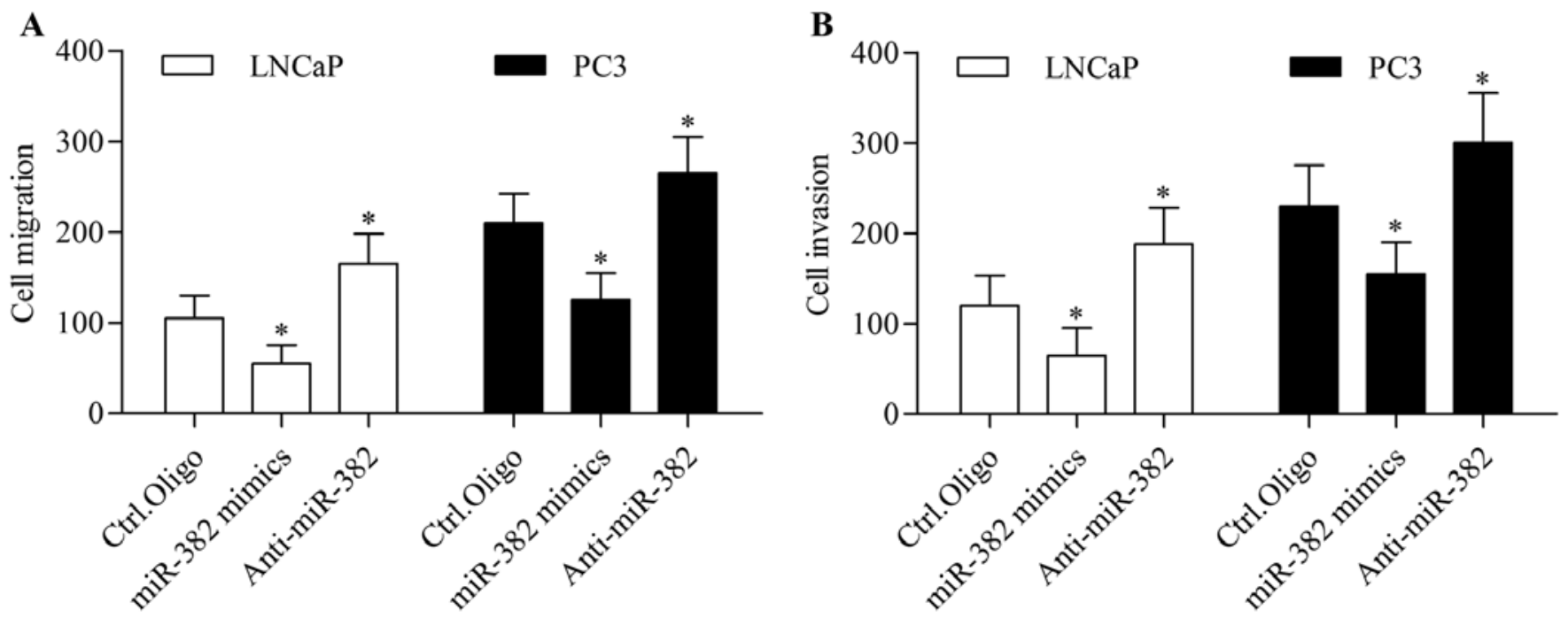

Figure 3. miR-382 inhibits prostate cancer cell migration and invasion. The effect of miR-382 mimics and anti-miR-382 on cell migration (A) and invasion (B) of LNCaP and PC3 cells was detected by Transwell assay. Migrated or invaded cells were stained by crystal violet, and cell number was counted under the microscope. "p<0.05 vs. Ctrl.Oligo.

mRNA targets of miR-382 and identified COUP-TFII as a putative target gene of miR-382 (Fig. 4A). To confirm the direct binding between miR-382 and the 3'-UTR of
COUP-TFII, we performed dual-luciferase reporter assay. The 3'-UTR of COUP-TFII containing complementary sequences to miR-382 seed sequences was cloned into a reporter vector. 
A

3'-gcuUAGGUGGUGCUUGUUGAAg-5' Hsa-miR-382

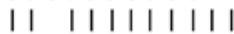

5'-uguGUCGGUCA--GAACAACUUg-3' COUP-TFII (WT)

5'-uguGUCGGUCA--GCAUACCGUg-3' COUP-TFII (MT)

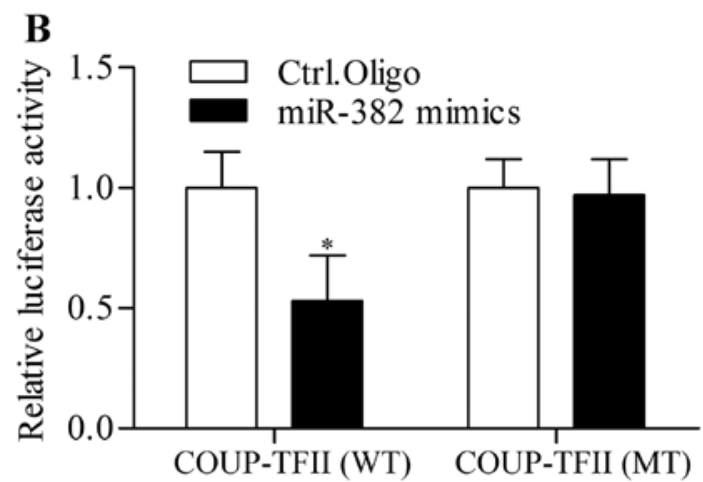

Figure 4. miR-382 targets COUP-TFII 3'-UTR. (A) Sequences of miR-382 and its seed sequence in the 3'-UTR of COUP-TFII. (B) The 3'-UTR of COUP-TFII linked luciferase activity assay. The wild-type (WT) or mutant (MT) luciferase reporter construct was transfected into 293T cells with miR-382 mimics. After incubation for $48 \mathrm{~h}$, the luciferase activity was detected by dual-Glo luciferase assay system. ${ }^{*} \mathrm{p}<0.05$ vs. Ctrl.Oligo.
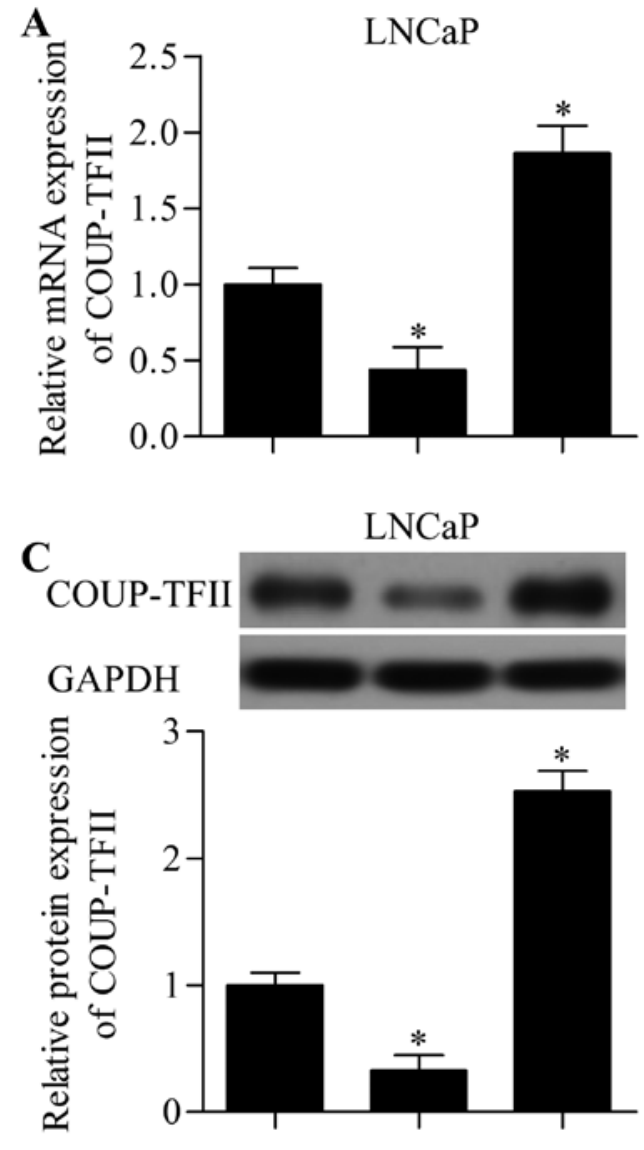

$\mathrm{LNCaP}$

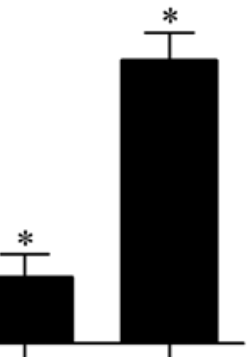

$\mathrm{LNCaP}$

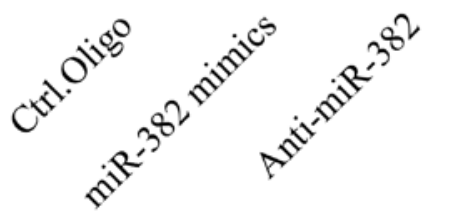

Cotransfection of the wild-type (WT) COUP-TFII 3'-UTR construct with miR-382 mimics into $293 \mathrm{~T}$ cells resulted in a significant decrease in luciferase activity compared with control (Fig. 4B). However, mutation of the predicated matching sites in the 3'-UTR of COUP-TFII (MT) significantly abrogated the suppressive effect of miR-382 mimics on luciferase activity (Fig. 4B). This result indicates that miR-382 directly targets the 3'-UTR of COUP-TFII. To assess whether miR-382 regulates COUP-TFII expression, we detected the effect of miR-382 mimics or anti-miR-382 on COUP-TFII expression by RT-qPCR and western blot analysis in LNCaP and PC 3 cells. RT-qPCR analysis showed that miR-382 overexpression significantly inhibited the mRNA expression level of COUP-TFII in LNCaP (Fig. 5A) and PC3 (Fig. 5B) cells. Furthermore, western blot analysis showed that miR-382 overexpression markedly suppressed the protein expression of COUP-TFII in LNCaP (Fig. 5C) and PC3 (Fig. 5D) cells. In contrast, miR-382 suppression significantly upregulated the mRNA and protein expression of COUP-TFII (Fig. 5). Taken together, these results suggest that miR-382 directly targets the 3'-UTR of COUP-TFII and regulates COUP-TFII expression in prostate cancer cells.
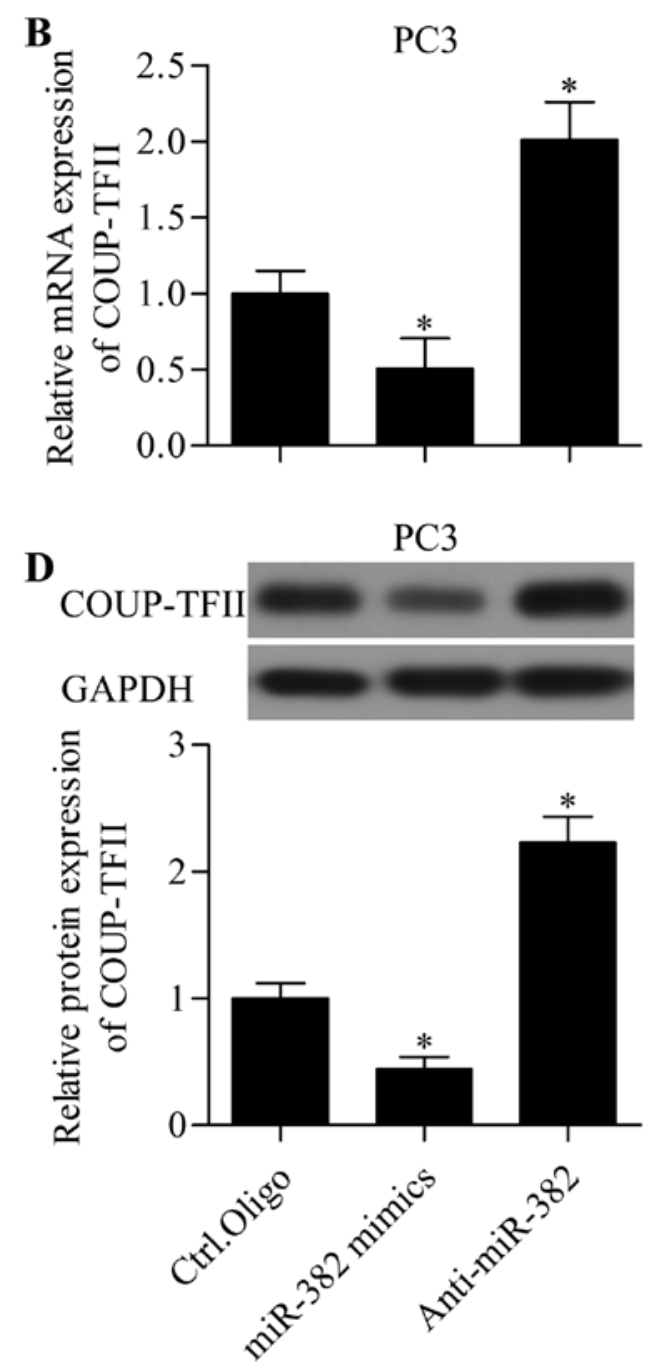

Figure 5. miR-382 inhibits COUP-TFII expression. LNCaP and PC3 cells were transfected with miR-382 mimics or anti-miR-382 for 48 h and harvested for analysis. The mRNA expression of COUP-TFII in LNCaP (A) and PC3 (B) cells was detected by RT-qPCR. The protein expression of COUP-TFII in LNCaP (C) and PC3 (D) cells was detected by western blot analysis. " $\mathrm{p}<0.05$ vs. Ctrl.Oligo. 
A

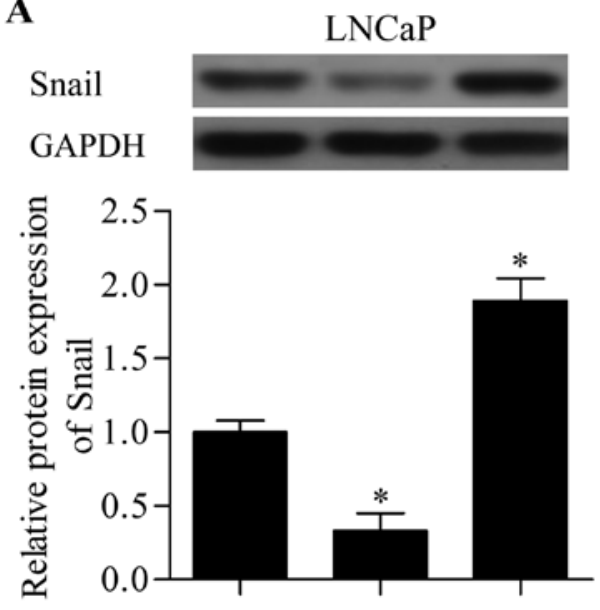

B
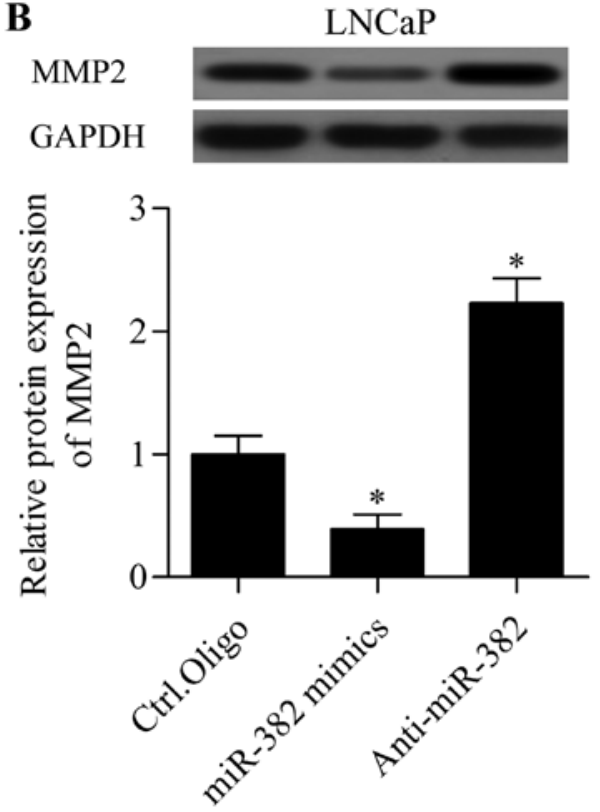

C
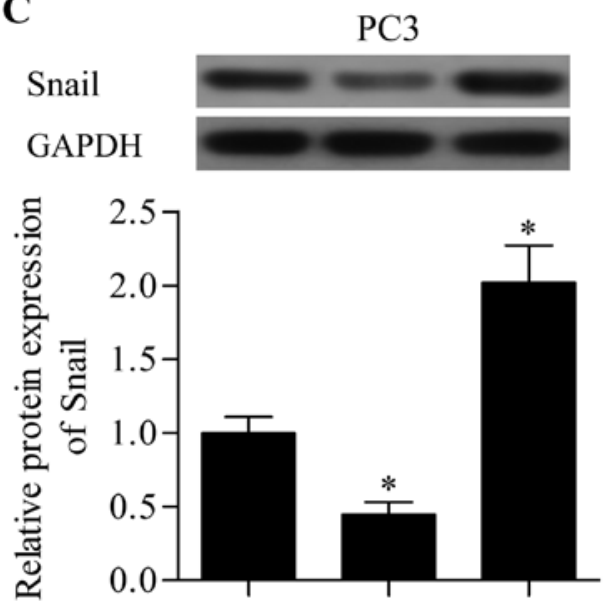

D
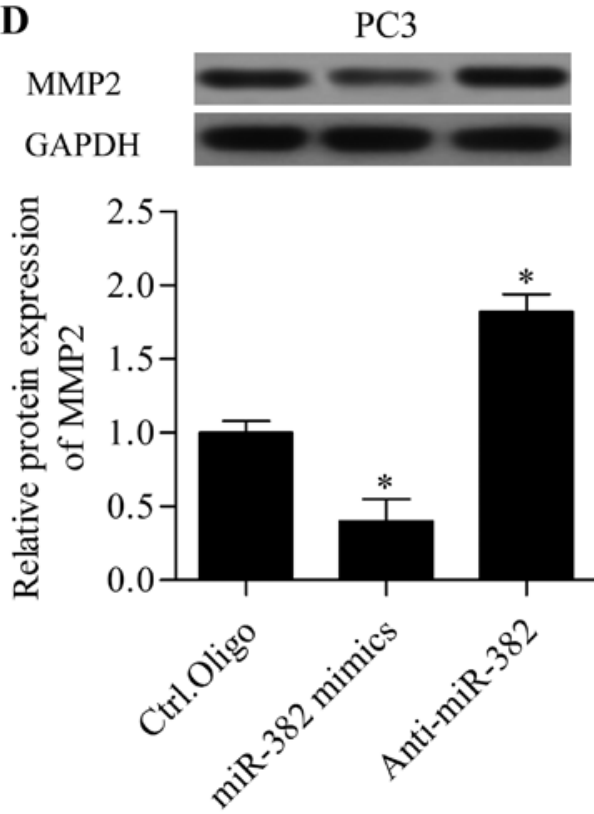

Figure 6. miR-382 inhibits Snail and MMP2 expression. Western blot analysis of Snail (A) and MMP2 (B) in LNCaP cells and Snail (C) and MMP2 (D) in PC3 cells. Cells were transfected with miR-382 mimics or anti-miR-382 for $48 \mathrm{~h}$ before being subjected to western blot analysis. "p<0.05 vs. Ctrl.Oligo.

miR-382 inhibits Snail and MMP2 expression. To further understand the molecular basis of miR-382 in regulating prostate cancer, we detected the regulatory effect of miR-382 on COUP-TFII downstream genes, including Snail and MMP2. Our data showed that miR-382 overexpression significantly inhibited the protein expression of Snail (Fig. 6A) and MMP2 (Fig. 6B) in LNCaP cells transfected with miR-382 mimics. Similarly, miR-382 overexpression markedly suppressed the protein expression of Snail (Fig. 6C) and MMP2 (Fig. 6D) in PC3 cells. However, miR-382 suppression exhibited opposite effect. These data indicate that miR-382 regulates Snail and MMP2 expression.

miR-382 functions via COUP-TFII. To verify whether miR-382 functions via COUP-TFII in prostate cancer, we detected the restoration of COUP-TFII on the suppressive effect of miR-382 by rescue experiments. We cotransfected miR-382 mimics with COUP-TFII expressing vector harboring no 3'-UTR into LNCaP and PC3. Results showed that COUP-TFII expressing vector transfection significantly restored the decreased COUP-TFII expression induced by miR-382 mimics (Fig. 7A and B). Furthermore, the decreased Snail expression induced by miR-382 overexpression was also significantly reversed by the restoration of COUP-TFII (Fig. 7C and D). In addition, the inhibitory effect of miR-382 on prostate cancer proliferation (Fig. 8A), migration (Fig. 8B), and invasion (Fig. 8C) was significantly reversed by the restoration of COUP-TFII. Taken together, these results imply that miR-382 represses prostate cancer proliferation and metastasis via COUP-TFII.

\section{Discussion}

We investigated the function of miR-382 in prostate cancer, and showed that miR-382 expression was significantly decreased in specimens from prostate cancer patients, as well as in prostate cancer cell lines. Interestingly, the overexpression of miR-382 inhibited prostate cancer cell proliferation, migration, and invasion, implying a tumor suppressor function of miR-382. 
A
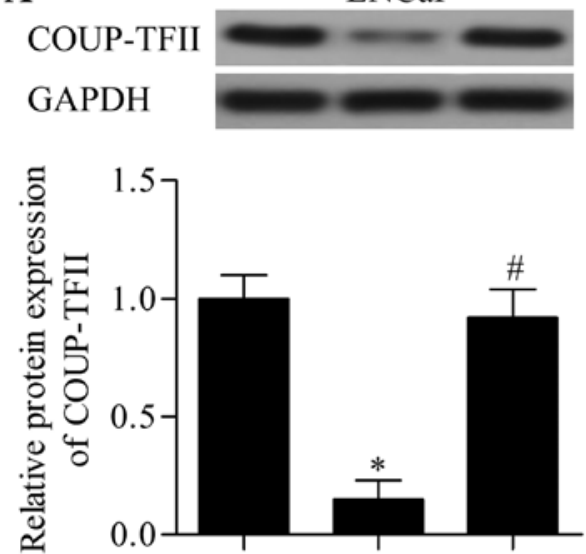

C

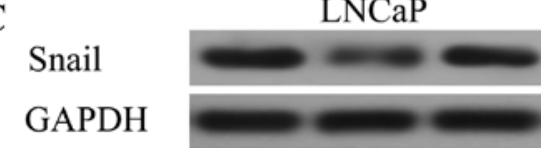

음

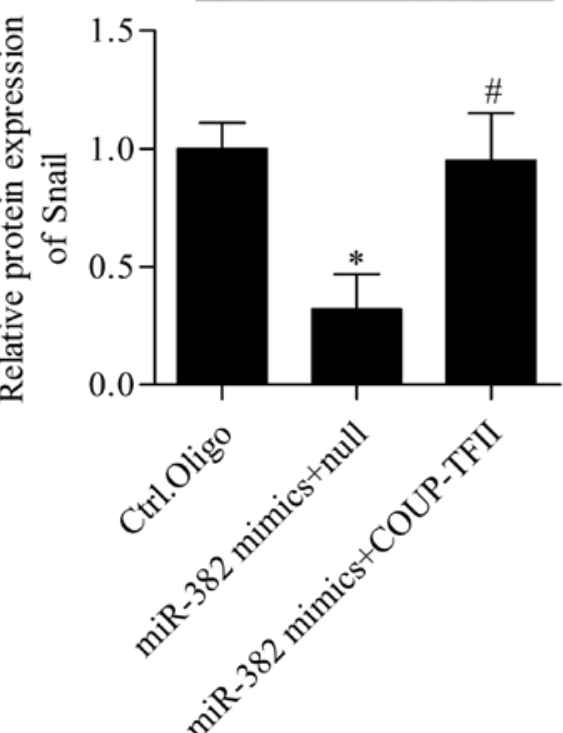

B
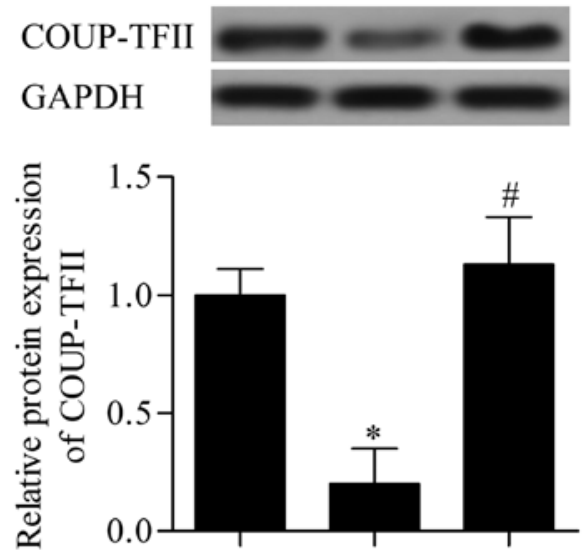

D

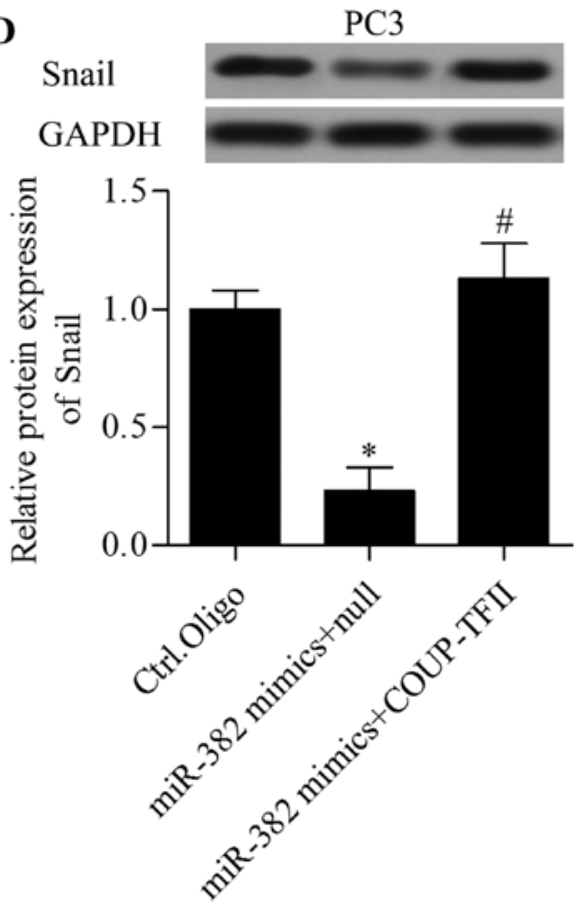

Figure 7. Restoration of COUP-TFII expression. Prostate cancer cells were cotransfected with miR-382 mimics and COUP-TFII expressing vector without 3'-UTR region and incubated for $48 \mathrm{~h}$. Null vector was used as control. Western blot analysis of COUP-TFII expression in LNCaP (A) and PC3 (B) cells. Western blot analysis of Snail expression in $\mathrm{LNCaP}(\mathrm{C})$ and PC3 (D) cells. ${ }^{*} \mathrm{p}<0.05$ vs. Ctrl.Oligo; ${ }^{\#}$ p $<0.05$ vs. miR-382 mimics+null.

A
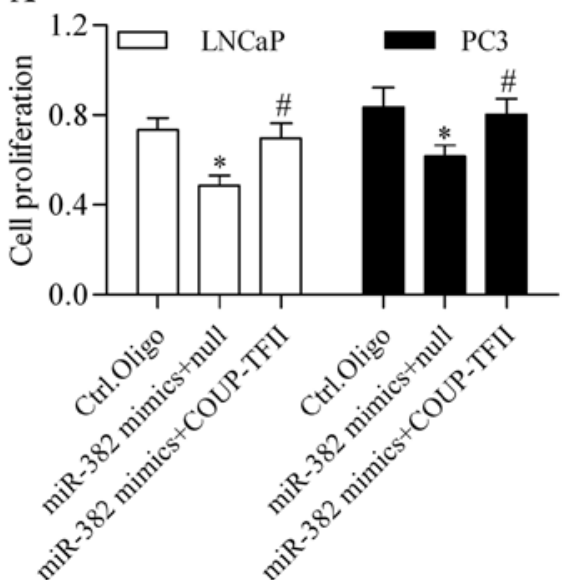

B
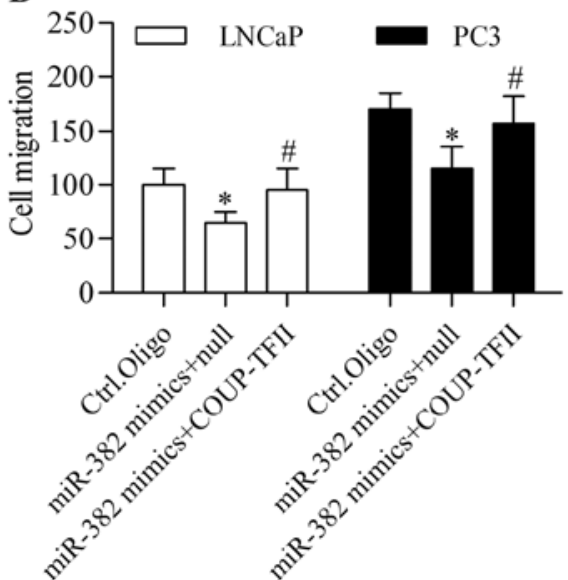

C
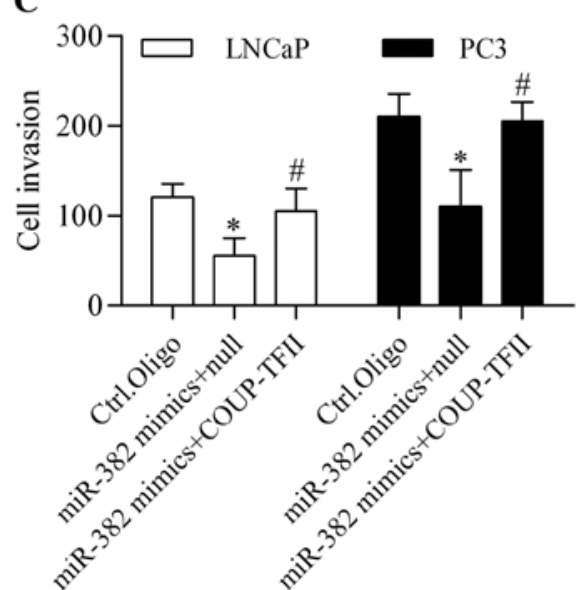

Figure 8. miR-382 functions through COUP-TFII. The effect of COUP-TFII restoration on cell proliferation (A), migration (B), and invasion (C) in LNCaP and PC 3 cells transfected with miR-382 mimics was determined. " $\mathrm{p}<0.05$ vs. Ctrl.Oligo; ${ }^{*} \mathrm{p}<0.05$ vs. miR-382 mimics+null. 
Further results revealed that COUP-TFII was verified as a target gene of miR-382, through which miR-382 regulated prostate cancer cell proliferation and metastasis. These results suggest an important function of miR-382 in prostate cancer.

A recent study demonstrates that the decreased miR-382 is associated with poor outcome in esophageal squamous cell cancer patients (24). In primary ovarian tumors, miR-382 is significantly decreased and may function through targeting the oncogene kinesin family member 14 (26). Tan and colleagues found that miR-382 was downregulated in human ovarian cancer tissues and cell lines; moreover, they found that miR-382 could inhibit proliferation, epithelial-mesenchymal transition, migration, and invasion of ovarian cancer cells (23). Xu et al reported that miR-382 is decreased in highly metastatic osteosarcoma cell lines and that the overexpression of miR-382 suppressed proliferation, epithelial-mesenchymal transition, and metastasis and enhanced chemo-sensitivity in osteosarcoma through targeting $\mathrm{Y}$ box-binding protein 1, Krüppel-like factor 12 , and homeodomain interacting protein kinase $3(25,27)$. miR-382 is found to be decreased in aggressive primary melanoma and miR-382 overexpression inhibits tumor cell metastasis in vitro and in vivo through targeting cortactin (28). Consistent with these findings, we found that miR-382 was significantly decreased in prostate cancer tissues and cell lines. The overexpression of miR-382 inhibited prostate cancer cell proliferation, migration, and invasion, indicating a tumor suppressor function of miR-382. Further mechanistic study revealed that miR-382 functioned through targeting COUP-TFII, which regulated cancer cell proliferation and metastasis. Interestingly, a more recent study reported that, in line with our findings, miR-382 suppresses colorectal cancer growth and invasion through targeting COUP-TFII (22). Although these reports and our study support the notion that miR-382 functions as a tumor suppressor, the contrary function of miR-382 is also reported. Seok et al reported that miR-382 induced by hypoxia promoted tumor growth and angiogenesis by inhibiting tumor suppressor phosphatase and tensin homolog (PTEN) in gastric cancer, supporting an oncogenic function of miR-382 (29). Similarly, Bei et al demonstrated that miR-382 promotes hepatocyte proliferation and cell growth by targeting PTEN signaling (30). These differences indicate that miR-382 may exert its function in a context-dependent manner among various cancer types.

COUP-TFII has been suggested as an important regulator in cancers, especially in prostate cancer (16). Increased expression of COUP-TFII is found in breast, ovarian, and colon cancers (31-33). High expression of COUP-TFII, which is found in prostate cancer, is associated with earlier tumor recurrences and decreased survival after prostatectomy $(20,34)$. COUP-TFII deficiency in PTEN-null mice prevents prostate cancer progression and cancer cell metastasis associated with TGF- $\beta /$ SMAD4-induced growth barrier (20). COUP-TFII regulates a series of downstream genes that is associated with cell proliferation, cell cycle, and metastasis $(18,33)$. Particularly, COUP-TFII can activate the transcription of the Snail, a key regulator of tumor metastasis (35), through which COUP-TFII are related to carcinogenesis and metastasis (18). Otherwise, the tumor suppressor function of COUP-TFII is also proposed (36-38). In this study, we have demonstrated that miR-382 could regulate Snail through
COUP-TFII, explaining the underlying mechanism of miR-382 in regulating prostate cancer cell proliferation and metastasis and supporting the tumor-promoter function of COUP-TFII. A recent study reveals that miR-101 and miR-27a inhibit the metastasis of prostate cancer by inhibiting COUP-TFII (21). In line with our study, the previous study elucidated that specific miRNAs contribute to prostate cancer progression and metastasis through the regulation of the COUP-TFII.

miRNAs represent the novel therapeutics for cancer. Besides miR-382 (22), miR-101, and miR-27a (21), other miRNAs can target COUP-TFII. miR-302 regulates stem cell differentiation through directly targeting COUP-TFII $(39,40)$. Kang et al reported that miR-302a promotes osteoblastic differentiation by repressing COUP-TFII (41). Similarly, miR-194 is reported to promote osteogenesis and inhibit adipogenesis by regulating COUP-TFII (42). Taken together, these reports offer a possibility of targeting COUP-TFII by specific miRNAs to treat diseases.

In conclusion, we show that miR-382 expression is decreased in prostate cancer. The overexpression of miR-382 significantly suppresses prostate cancer cell proliferation, migration, and invasion directly through targeting COUP-TFII. Thus, miR-382 may function as a tumor suppressor in prostate cancer. Our study suggests that miR-382 and COUP-TFII are potential and promising molecular targets for prostate cancer therapy.

\section{References}

1. Siegel RL, Miller KD and Jemal A: Cancer statistics, 2016. CA Cancer J Clin 66: 7-30, 2016.

2. Coleman RE: Clinical features of metastatic bone disease and risk of skeletal morbidity. Clin Cancer Res 12: S6243-S6249, 2006.

3. Mendell JT and Olson EN: MicroRNAs in stress signaling and human disease. Cell 148: 1172-1187, 2012.

4. Bartel DP: MicroRNAs: Genomics, biogenesis, mechanism, and function. Cell 116: 281-297, 2004.

5. Winter J, Jung S, Keller S, Gregory RI and Diederichs S: Many roads to maturity: microRNA biogenesis pathways and their regulation. Nat Cell Biol 11: 228-234, 2009.

6. Esquela-Kerscher A and Slack FJ: Oncomirs - microRNAs with a role in cancer. Nat Rev Cancer 6: 259-269, 2006.

7. Cho WC: OncomiRs: The discovery and progress of microRNAs in cancers. Mol Cancer 6: 60, 2007.

8. Ranganathan K and Sivasankar V: MicroRNAs - Biology and clinical applications. J Oral Maxillofac Pathol 18: 229-234, 2014.

9. Wang YL, Wu S, Jiang B, Yin FF, Zheng SS and Hou SC: Role of microRNAs in prostate cancer pathogenesis. Clin Genitourin Cancer 13: 261-270, 2015

10. Wang LH, Tsai SY, Cook RG, Beattie WG, Tsai MJ and O'Malley BW: COUP transcription factor is a member of the steroid receptor superfamily. Nature 340: 163-166, 1989.

11. Tang K, Rubenstein JL, Tsai SY and Tsai MJ: COUP-TFII controls amygdala patterning by regulating neuropilin expression. Development 139: 1630-1639, 2012.

12. Li L, Xie X, Qin J, Jeha GS, Saha PK, Yan J, Haueter CM, Chan L, Tsai SY and Tsai MJ: The nuclear orphan receptor COUP-TFII plays an essential role in adipogenesis, glucose homeostasis, and energy metabolism. Cell Metab 9: 77-87, 2009.

13. You LR, Lin FJ, Lee CT, DeMayo FJ, Tsai MJ and Tsai SY: Suppression of Notch signalling by the COUP-TFII transcription factor regulates vein identity. Nature 435: 98-104, 2005.

14. Yu CT, Tang K, Suh JM, Jiang R, Tsai SY and Tsai MJ: COUP-TFII is essential for metanephric mesenchyme formation and kidney precursor cell survival. Development 139: 2330-2339, 2012.

15. Qin J, Chen X, Xie X, Tsai MJ and Tsai SY: COUP-TFII regulates tumor growth and metastasis by modulating tumor angiogenesis. Proc Natl Acad Sci USA 107: 3687-3692, 2010. 
16. Qin J, Tsai SY and Tsai MJ: The critical roles of COUP-TFII in tumor progression and metastasis. Cell Biosci 4: 58, 2014.

17. Navab R, Gonzalez-Santos JM, Johnston MR, Liu J, Brodt P, Tsao MS and Hu J: Expression of chicken ovalbumin upstream promoter-transcription factor II enhances invasiveness of human lung carcinoma cells. Cancer Res 64: 5097-5105, 2004.

18. Bao Y, Gu D, Feng W, Sun X, Wang X, Zhang X, Shi Q, Cui G, $\mathrm{Yu} \mathrm{H}$, Tang C, et al: COUP-TFII regulates metastasis of colorectal adenocarcinoma cells by modulating Snaill. Br J Cancer 111: 933-943, 2014

19. Polvani S, Tarocchi M, Tempesti S, Mello T, Ceni E, Buccoliero F, D'Amico M, Boddi V, Farsi M, Nesi S, et al: COUP-TFII in pancreatic adenocarcinoma: Clinical implication for patient survival and tumor progression. Int J Cancer 134: 1648-1658, 2014.

20. Qin J, Wu SP, Creighton CJ, Dai F, Xie X, Cheng CM, Frolov A, Ayala G, Lin X, Feng XH, et al: COUP-TFII inhibits TGF- $\beta$ induced growth barrier to promote prostate tumorigenesis. Nature 493: 236-240, 2013.

21. Lin SC, Kao CY, Lee HJ, Creighton CJ, Ittmann MM, Tsai SJ, Tsai SY and Tsai MJ: Dysregulation of miRNAs-COUP-TFII -FOXM1-CENPF axis contributes to the metastasis of prostate cancer. Nat Commun 7: 11418, 2016.

22. Zhou B, Song J, Han T, Huang M, Jiang H, Qiao H, Shi J and Wang Y: MiR-382 inhibits cell growth and invasion by targeting NR2F2 in colorectal cancer. Mol Carcinog: Jan 22, 2016 (Epub ahead of print). doi: $10.1002 / \mathrm{mc} .22466$.

23. Tan H, He Q, Gong G, Wang Y, Li J, Wang J, Zhu D and Wu X miR-382 inhibits migration and invasion by targeting ROR through regulating EMT in ovarian cancer. Int J Oncol 48 : 181-190, 2016

24. Qi B, Lu JG, Yao WJ, Chang TM, Qin XG, Ji YH, Wang TY, Liu SG, Li HC, Liu YZ, et al: Downregulation of microRNA-382 is associated with poor outcome of esophageal squamous cell carcinoma. World J Gastroenterol 21: 6884-6891, 2015.

25. Xu M, Jin H, Xu CX, Sun B, Mao Z, Bi WZ and Wang Y: miR-382 inhibits tumor growth and enhance chemosensitivity in osteosarcoma. Oncotarget 5: 9472-9483, 2014.

26. Thériault BL, Basavarajappa HD, Lim H, Pajovic S, Gallie BL and Corson TW: Transcriptional and epigenetic regulation of KIF14 overexpression in ovarian cancer. PLoS One 9: e91540, 2014.

27. Xu M, Jin H, Xu CX, Sun B, Song ZG, Bi WZ and Wang Y: miR-382 inhibits osteosarcoma metastasis and relapse by targeting Y box-binding protein 1. Mol Ther 23: 89-98, 2015.

28. Hanniford D, Segura MF, Zhong J, Philips E, Jirau-Serrano X, Darvishian F, Berman RS, Shapiro RL, Pavlick AC, Brown B, et al: Identification of metastasis-suppressive microRNAs in primary melanoma. J Natl Cancer Inst 107: 107, 2015.

29. Seok JK, Lee SH, Kim MJ and Lee YM: MicroRNA-382 induced by HIF-1 $\alpha$ is an angiogenic miR targeting the tumor suppressor phosphatase and tensin homolog. Nucleic Acids Res 42: 8062-8072, 2014.

30. Bei Y, Song Y, Wang F, Dimitrova-Shumkovska J, Xiang Y, Zhao Y, Liu J, Xiao J and Yang C: miR-382 targeting PTEN-Akt axis promotes liver regeneration. Oncotarget 7: 1584-1597, 2016.
31. Nagasaki S, Suzuki T, Miki Y, Akahira J, Shibata H, Ishida T, Ohuchi N and Sasano H: Chicken ovalbumin upstream promoter transcription factor II in human breast carcinoma: Possible regulator of lymphangiogenesis via vascular endothelial growth factor-C expression. Cancer Sci 100: 639-645, 2009.

32. Shin SW, Kwon HC, Rho MS, Choi HJ, Kwak JY and Park JI: Clinical significance of chicken ovalbumin upstream promotertranscription factor II expression in human colorectal cancer. Oncol Rep 21: 101-106, 2009.

33. Hawkins SM, Loomans HA, Wan YW, Ghosh-Choudhury T, Coffey D, Xiao W, Liu Z, Sangi-Haghpeykar H and Anderson ML: Expression and functional pathway analysis of nuclear receptor NR2F2 in ovarian cancer. J Clin Endocrinol Metab 98: E1152-E1162, 2013.

34. Qin J, Tsai S and Tsai MJ: COUP-TFII, a prognostic marker and therapeutic target for prostate cancer. Asian J Androl 15: 360-361, 2013.

35. Kudo-Saito C, Shirako H, Takeuchi T and Kawakami Y: Cancer metastasis is accelerated through immunosuppression during Snail-induced EMT of cancer cells. Cancer Cell 15: 195-206, 2009.

36. Song CH, Lee HJ, Park E and Lee K: The chicken ovalbumin upstream promoter-transcription factor II negatively regulates the transactivation of androgen receptor in prostate cancer cells PLoS One 7: e49026, 2012.

37. Litchfield LM, Appana SN, Datta S and Klinge CM: COUP-TFII inhibits NFkappaB activation in endocrine-resistant breast cancer cells. Mol Cell Endocrinol 382: 358-367, 2014.

38. Nakshatri H, Mendonca MS, Bhat-Nakshatri P, Patel NM, Goulet RJ Jr and Cornetta K: The orphan receptor COUP-TFII regulates $\mathrm{G} 2 / \mathrm{M}$ progression of breast cancer cells by modulating the expression/activity of $\mathrm{p} 21$ (WAF1/CIP1), cyclin D1, and cdk2. Biochem Biophys Res Commun 270: 1144-1153, 2000.

39. Rosa A and Brivanlou AH: A regulatory circuitry comprised of miR-302 and the transcription factors OCT4 and NR2F2 regulates human embryonic stem cell differentiation. EMBO J 30: 237-248, 2011.

40. Hu S, Wilson KD, Ghosh Z, Han L, Wang Y, Lan F, Ransohoff KJ, Burridge $\mathrm{P}$ and $\mathrm{Wu}$ JC: MicroRNA-302 increases reprogramming efficiency via repression of NR2F2. Stem Cells 31: 259-268, 2013.

41. Kang IH, Jeong BC, Hur SW, Choi H, Choi SH, Ryu JH, Hwang YC and Koh JT: MicroRNA-302a stimulates osteoblastic differentiation by repressing COUP-TFII expression. J Cell Physiol 230: 911-921, 2015.

42. Jeong BC, Kang IH, Hwang YC, Kim SH and Koh JT: MicroRNA-194 reciprocally stimulates osteogenesis and inhibits adipogenesis via regulating COUP-TFII expression. Cell Death Dis 5: e1532, 2014. 